\title{
Treatment of Clavicle Fractures in Athletes and Young Active Patients
}

\author{
Ulucay Cagatay*, Faik Altintas, Turhan Ozler and Burak Aksu
}

Yeditepe Medical Faculty Istanbul, Turkey

\begin{abstract}
Clavicle fractures are very common and nearly all clavicle fractures heal with simple conservative methods. Clavicle fractures are also rather frequent in athletes as well as the ordinary young, active people because of simple falls and accidents. In this review, current treatment modalities for clavicle fractures in athletes and young active patients are given briefly. Those suggestions may also be beneficial for a person seeking for a faster recovery.
\end{abstract}

Keywords: Shoulder girdle; Clavicle; Locked plate; Fracture

\section{Introduction}

Clavicle fractures are known since the beginning of mankind. Although it is very common, nearly all heals with simple conservative methods. That may be the reason why it is neglected for a long time. Now, we know that clavicle is an important bone for the shoulder girdle and its movements. Maintaining the length, rotation, and alignment of clavicle is important especially for an upper extremity performing elite athlete to continue his/hers skills and abilities after a fracture. This review is based on treatment of clavicular fractures especially for an athlete and a young active patient seeking for a faster recovery.

\section{Fractures of the Clavicle}

Clavicle fractures are one of the most frequent fractures; nearly $2 \%$ to $5 \%$ of all fractures in adults and $10 \%$ to $15 \%$ in children, and $35 \%$ of injuries of the shoulder girdle involve clavicle [1-5]. Fractures of the clavicle also show a bimodal age distribution as young male patients less than 30 years of age and elderly patients over 70 years of age [4]. In adults, more than $70 \%$ of these fractures occur at the midshaft of the clavicle, and these injuries are more likely to be displaced as compared with medial and lateral third fractures. In children, up to $90 \%$ of clavicle fractures are midshaft fractures $[1,3,4,6]$. Medial third accounts for $2-3 \%$ and lateral third for $25-28 \%[1,3,4]$.

There are several classification systems in the literature. In this review, the given classification is Edinburgh Classification. This classification is rather simple, accepted with satisfactory levels of interobserver variation for reliability, and maintains similar outcome results [5]. This classification based on the anatomic location, displacement, comminution, and articular involvement. Figure 1 is an illustration of this this classification.

Traditionally, nonsurgical management has been favored as the initial treatment modality for most clavicle fractures because high nonunion rates had been reported after operative treatment in the literature $[7,8]$. We know that conservative treatment may come with its burden as long immobilization period, malunion, clavicle shortening, muscle weakness, and cosmetic problems $[9,10]$. Since the main concern of this review is treatment of athletes and young adults, more rapid healing and returning back to sports will be especially given for treatment type. It does not mean plating or nailing every clavicle crack. In Figure 2, a successful union of a comminuted clavicle fracture treated with conservative methods is also given. Never forget that nearly all clavicle fractures unite with conservative treatment and dealing with an infected non-union of a previously operated clavicle is one of the worst scenarios an orthopedic surgeon may experience.

The clavicle fractures can result from either an indirect trauma with a fall to an outstretched arm or, much less commonly, they occur with a direct blow to the clavicle. For adults the most common causes are MVC, sports injuries, fall, and direct trauma [2,11,12]. For sports injuries, indirect traumas are more common but in football, rugby, cycling, or skiing; fractures may happen with direct traumas. Accepted emergent operative treatment for clavicle fractures are open fractures, accompanying neurovascular injury, and floating shoulder [11].

Clavicular fractures generally produce an obvious painful deformity, with local tenderness over the fracture. There is often downward displacement of the lateral fragment of the fractured clavicle under the weight of the shoulder and elevation of the medial fragment by pull of the sternocleidomastoid muscle. Prominent sharp end of the medial fragment could produce a buttonhole at the skin in many cases. In rare cases, there could be a compression of the mediastinum, or the major vessels with the posterior displacement of the medial fragment of the clavicle. The whole arm distal to the fracture should

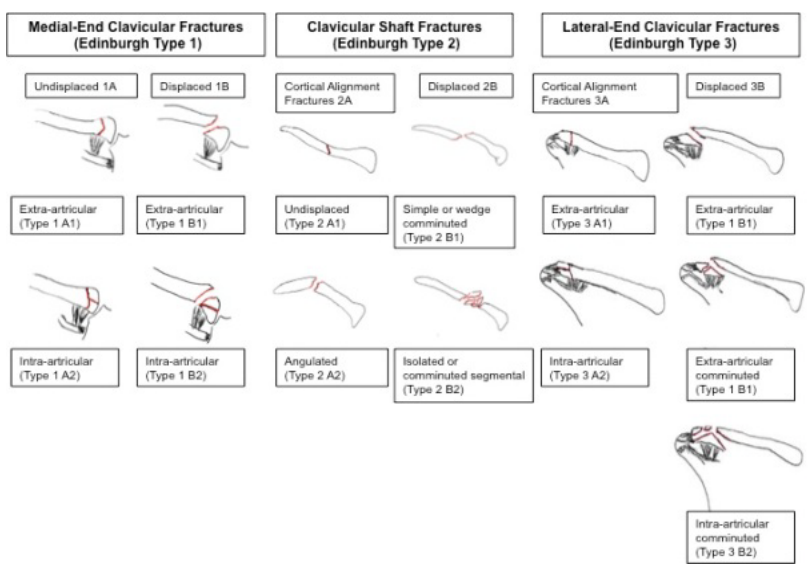

Figure 1: Illustration of Edinburgh classification

*Corresponding author: Ulucay Cagatay, Yeditepe Medical Faculty, Orthopaedics and Traumatology, Devlet yolu Ankara caddesi no 102 Kozyatagi, Istanbul, Turkey, Tel: 905327284719; E-mail: culucay@turk.net

Received January 14, 2015; Accepted February 24, 2015; Published February 26, 2015

Citation: Cagatay U, Altintas F, Ozler T, Aksu B (2015) Treatment of Clavicle Fractures in Athletes and Young Active Patients. J Trauma Treat 4: 237. doi:10.4172/2167-1222.1000237

Copyright: (c) 2015 Cagatay U, et al. This is an open-access article distributed under the terms of the Creative Commons Attribution License, which permits unrestricted use, distribution, and reproduction in any medium, provided the original author and source are credited. 


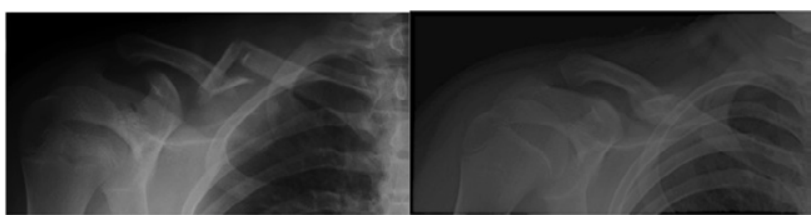

Figure 2: A successful union of a comminuted clavicle fracture treated with conservative methods.

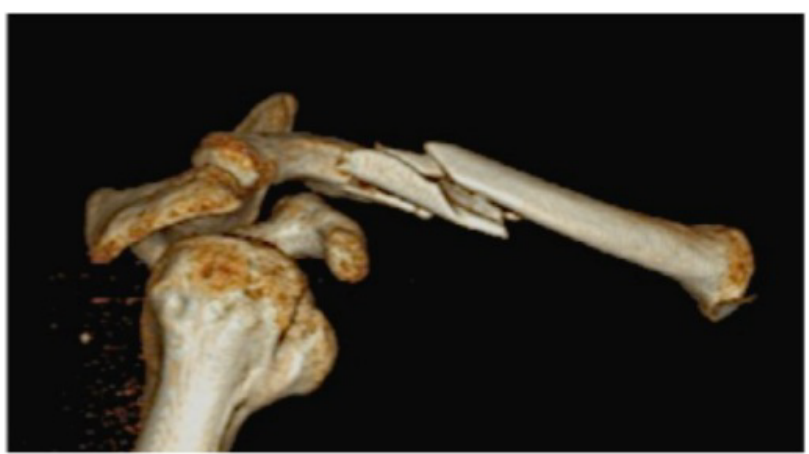

Figure 3: 3D CT of a comminuted clavicle fracture.

be examined for brachial plexus or vascular injury. Neurovascular injury may happen either directly by displaced fracture fragments or by stretching injuries. A difference in blood pressure between the two upper extremities suggests a vascular injury, and arterial doppler or arteriography should be done if there is a suspicion.

The radiographic diagnosis is usually made on a single anteroposterior shoulder view. In the literature, posteroanterior $15^{\circ}$ caudad view radiograph for determining the shortening of clavicle is also mentioned. Spiral computerized tomography (CT) gives the best results especially for a preoperative planning of a comminuted fracture [13]. In Figure 3, a 3D CT of a comminuted clavicle fracture is given. Complete chest and rib examination also needed in clavicle fractures. It is not uncommon a clavicle fracture complicated with pneumothorax, hemothorax or flair chest $[4,6]$.

\section{Treatment of Medial-end Clavicular Fractures (Edinburgh Type 1)}

Fractures of the medial third of clavicle are rare, and nearly always non/minimal displaced, and nearly all treated conservatively. A simple a shoulder sling or strap between 2-6 weeks is enough for treatment [4]. The main aim of the treatment is patient's comfort and pain reduction. Early range of motion is encouraged. A structured rehabilitation ensures a satisfactory outcome for most patients. To protect the healing clavicle, it is important to avoid contact sports for a minimum of 2-5 months. Emergent surgical treatment is required when there is compression on mediastinal structures because of the posterior displacement. Stability of this fracture depends on the integrity of the costoclavicular ligament. If it is torn, the lateral fragment displaces anteriorly and may overlap the medial fragment. Either closed or open reduction under general anesthesia has to be performed. After the adequate reduction, the fixation can be done with plates, Balser plate, interosseous sutures or screws $[6,14]$. The use of unthreaded pins or K-wires are dangerous, and there are many complications of migration to vital organs in the literature $[3,4]$. For the young patients, it has to be kept in mind that medial epiphysis of the clavicle does not ossify up to 18 years age and does not fuse before 25 years of age [15]. It is important to differentiate sternoclavicular joint (SCJ) dislocation with type 1 fracture because of the remodelling potential. For those cases a CT or an MRI is a better choice for screening.

\section{Treatment of Shaft Fractures (Edinburgh Type 2)}

There is general agreement that non-displaced fractures (type 2A) should be treated nonoperatively $[3,4,16]$. The traditional choice of the treatment of displaced fractures is also mainly conservative. For my belief, the reason this choice was the bad results of operative treatment such as high risk of non-union, implant irritation, and infection with old-fashioned (era) surgical methods and implants [7].

The main methods of conservative treatment are: Shoulder sling, which has some issues of stability and maintaining the reduction; strap, which may be responsible for axillary pressure sores, compression of the neurovascular bundle, and higher rates of nonunion; taping, which has low power for maintaining stability, skin problems, and difficulty in easing the pain. Unless which method you are using, the immobilization period depends for the acute pain's subsidence (like 3-6 weeks), and patients are encouraged to undertake normal activities as pain allows. Recovery of the range of motion and function of the shoulder is usually swift if the fracture unites, and sometimes physiotherapy required. Most patients respond well to self-administered program of rangeof-motion and muscle-strengthening exercises. The return to contact sports is about 4-6 months.

After a mid-shaft of clavicle in an elite athlete or an active young person, the main concern is when he/she can return to training, job and when will start contact sports and daily life. In nowadays, apart from the athletes, ordinary young people also require for a shorter time for returning back to sports and need their arm's power restored as before with less bad cosmetic results. Clavicle will heal with conservative methods but needs time. That's why especially for a young person who insists on returning back to sports or labor, surgical treatment has to be chosen. There are variety of methods could be chosen from surgical treatment. The orthopaedic surgeon has to keep in mind that stable fixation (absolute or relative stability) essential for a fast recovery.

\section{Plate fixation}

This technique provides immediate rigid stabilization and pain relief and facilitates early mobilization and somehow the golden method for operative treatment [3,4,12,16-19]. Currently, the most commonly used implants are dynamic compression, or locking plates. Reconstruction plates are not used anymore because of their weak resistance to deforming forces that leads to non/malunion. In Figure 4 , an implant failure and non-union of a clavicle fracture fixed with reconstruction plate is given. Site-specific precontoured locking

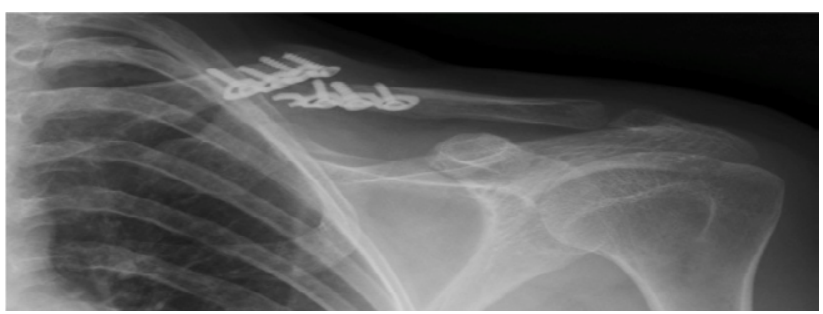

Figure 4: An implant failure and non-union of a clavicle fracture fixed with reconstruction plate. 




Figure 5: A successful union of a comminuted clavicle fracture, achieved withprecontoured locked plate.

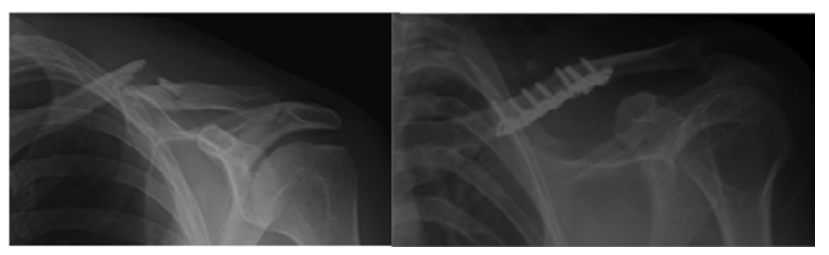

Figure 6: A successful union of a clavicle fracture, achieved with anterior locked plate.

plates have recently been on the market, which is popularized for less prominence under skin, and high resistance to deforming forces with locking screws $[11,20]$. Although their success rate is high, be aware of the complications which are infection, plate failures, skin irritation, keloid scars, non-union, and iatrogenic neurovascular injury [11]. Every company has some different sizes and shapes of plates in their tray. You have to choose which one is better fitted to the patient's clavicle's superior shape, and size. In Figure 5, a successful union of a comminuted clavicle fracture, achieved with precontoured locked plate is given. There are some investigations on going whether the shapes and the sizes of the plates in the market are suitable for every person or "person specific plates" are required. Also some companies start to produce plates, which are precontoured for anterior/antero-inferior contours of clavicle. Although the biomechanical testing is less secure than the superior position, lower neurovascular complication rates while implantation and similar union rates makes them also favorable $[11,21]$. In Figure 6, a successful union of a clavicle fracture, achieved with anterior locked plate is given. One new trend is percutaneous plating, which is getting popular. With this method, the facture site does not opened and provoked healing and shorter union time may benefitted [22]. In the surgery, care has to given for the main principles of fracture treatment such as protecting the soft tissue coverage, and periost, and anatomic reduction. Debates are still going on going whether using bone grafts, DBM, or putty in fresh fractures or just fixation is adequate $[23,24]$. Postoperative early motion is possible with stable fixation and only the surgeon considers "when the patient is ready to back to contact sports and labor" according to pain and union process.

\section{Intramedullary fixation}

Unlike the general Intra Medullary Nails (IMN) for the long bones, clavicle's sigmoid shape, and narrow medullary canal, make a proximal and distal locking nail design impossible. There are variety of intramedullary devices in the market such as K-wires, Knowles pins, Hangie pins, Rockwood pins, and elastic titanium intramedullary nail [25]. Although there is biomechanical evidence to suggest that plate fixation provides a stronger construct than intramedullary fixation, IMN gives a minimally invasive approach with biological healing, better cosmetic results, less periosteal stripping and less soft tissue destruction $[4,25]$. The most popular method is the new antegrade titanium nails, passed through an anteromedial entry point in the medial fragment. An entry hole has produced by awl with respecting the costoclavicular ligament and SCJ capsule. This could be done under image intensifier with closed or open manner. Although this is a rather simple technique, it may result with many complications. The most common one is telescoping and shortening of the clavicle after the operation especially in comminuted fractures since the nail is not static. High rates of implant breakage, temporary brachial plexus palsy, skin irritations, and delayed pain relief are the other known complications [4,6,17]. Newly published papers are mentioning IMN combination with MIPO plates for the treatment of comminuted fractures without telescoping and shortening of clavicle after the operation [22]. In Figure 7, treatment of a clavicle fracture with a new generation IMN is given.

\section{External fixators}

They are useful only in open fractures, massive skin irritations, temporary fixation for damage control, and infected non-unions [26]. Unilateral or semicircular fixators are suitable for the fixation. Meticulous care has to give for preventing iatrogenic neurovascular complications. They don't have a rational role in treatment of an acute simple shaft fractures

\section{Treatment of Lateral-End Clavicular Fractures (Edinburgh Type 3)}

Most of the lateral-end fractures of clavicle are minimally displaced and extraarticular. The coronoid and trapezoid ligaments, with the help of intact periosteum, holds the distal part non-displaced usually. Therefore, for the majority of cases, conservative methods are suitable for treatment. Using a shoulder sling or strap for a limited time is generally enough with successful results $[4,6,17]$. If the fracture is displaced (Type $3 \mathrm{~B}$ ); there is also a debate for choosing the treatment type. Although conservative treatment is favored with good results with less complication, most of the patients in those studies were mid aged sedentary people whom can handle some pain with minimal loss of function [6]. We have to know for an athlete or a young active person; they need a very well reconstructed shoulder girdle to perform their skills or labor. Therefore, operative treatment is more considerable for a young person especially involving with overhead activities. There are some methods, and implants on the market and none of them generally accepted for the golden standard for the operative treatment of this fracture [6,11,27-32].

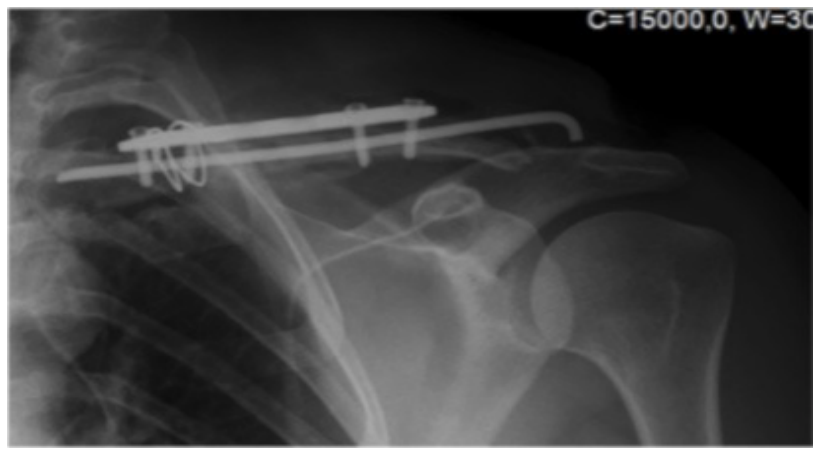

Figure 7: A successful union of a clavicle fracture, achieved with a combination if IMN pin with plates and cerclage wires. 


\section{Plate fixation}

An adequate stable plate fixation can be achieved only if the distal fragment is large enough to hold a minimum two bicortical screws [6]. Therefore in the past studies the non-union rates were considerably high because the fragments usually are small and comminuted in most of the cases. Those reconstruction or tubular plates cannot fix those small particles to the plate. Nowadays, many companies start to produce specific plates, which have multiple fixation holes with thin locked screws in the lateral end, and have regular locked screws for the shaft. Those kinds of plates may improve the stability with anatomic reduction [33]. In Figure 8, a successful union of a distal end a comminuted clavicle fracture, achieved with a new age distal clavicle locked plate is given. The clavicular hook plate was also developed for treatment of those fractures especially when the distal fragment is too small $[34,35]$. The plate has an offset lateral hook, designed to engage distal to the posterior aspect of the acromion. The results were favorable when it was compared to k-wires fixation [34]. The results may vary according to shoulder stiffness, acromioclavicular osteoarthritis, skin disturbance, osteolysis, and inadequate fixation with improper positioning of the plate [6]. Most surgeons advise routine plate removal at three months postoperatively for avoiding some of the complications but this may lead to non-union or refracture. Therefore, it is not a first choice of implant for an athlete or a young active person.

\section{Coracoclavicular screw}

The use of a coracoclavicular screw was first described in the treatment of acromioclavicular joint subluxation (Bosworth technique)

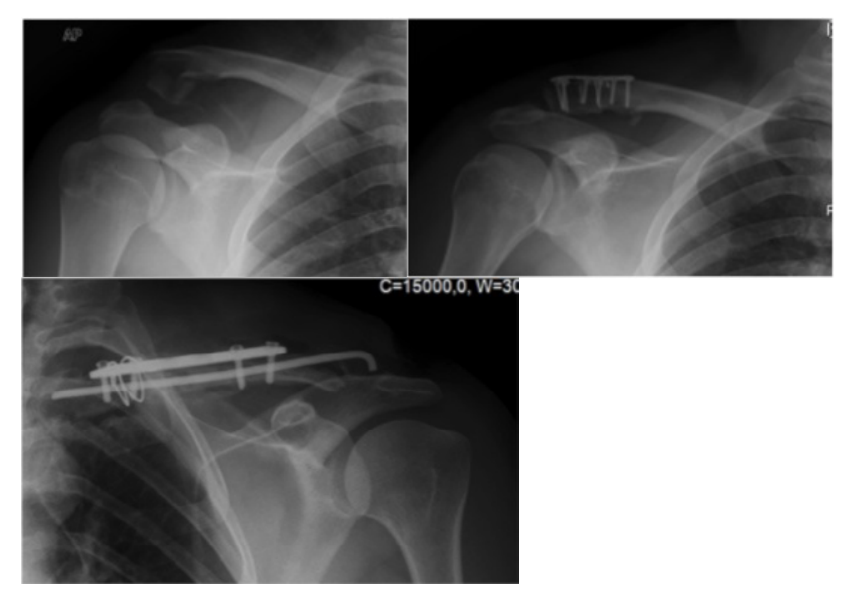

Figure 8: A successful union of a distal end a comminuted clavicle fracture, achieved with a new age distal clavicle locked plate.

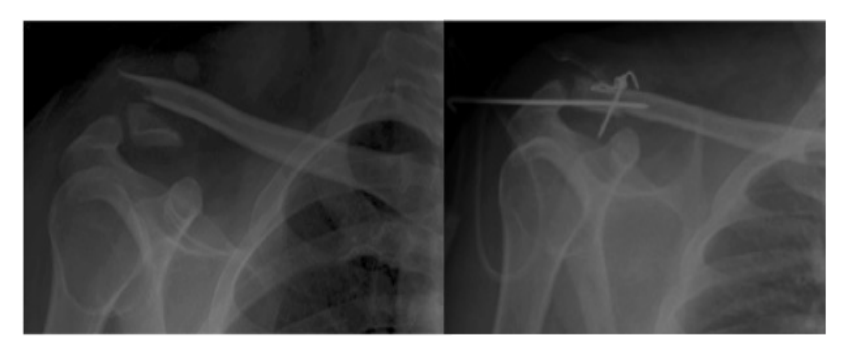

Figure 9: A successful union of a distal end a clavicle fracture, achieved k-wires combined with cerclage wire. and it is a technically demanding method that fixes the distal fragment to the coracoid process with a screw with/without a washer [36]. The results may vary in the literature and mainly investigated in small groups. Implant removal also needed afterwards. It can be used in some selected cases only regarding to high complication rate, and high technical demand.

\section{Kirschner wire fixation}

This technique was very popular in the late 60 's and this was the only choice a surgeon had at that time. In Figure 9, a successful union of a distal end a clavicle fracture, achieved k-wires combined with cerclage wire is given. The results may vary and complications are unpredictable starting from non-union ending with migration of the wire into vital organs and main vessels. Therefore, it is not a recommended choice of implant now.

\section{Suture and sling techniques}

Coracoclavicular sling and synthetic graft ligaments have been used to reconstruct the coracoclavicular ligaments in acromioclavicular joint (ACJ) dislocations, both as the primary stabilization and as reinforcement of other fixation techniques with good functional results [37-40]. Graft either is looped around the coracoid and over the fractured distal clavicle fragment to form a sling or passed through drill-holes. Instead of grafts, non-absorbable materials can also be used (Tightrope system (Arthrex, Naples, FL)). Fixation can be done either with endobuttons or suture anchors. With this technique, stable fixation without absolute rigidity can be maintained, and no implant removal operation is required. The fixation has enough stability for allowing early motion [37,41]. Arthroscopic ligament reconstruction techniques to treat displaced lateral-end fractures have recently been described and claim to have better functional results with less soft tissue disruption [30,41-43].

\section{Distal Clavicle Osteolysis (DCO)}

This is a phenomenon, which commonly results after either from a shoulder trauma, or a stress reaction to intolerable levels of exercise. Apart from the trauma cases, especially weightlifters are at risk. The patient complains a dull ache around ACJ and distal clavicle, weakness and some pain in flexion and abduction. Radiography may show some localized osteoporosis, tapering, and sometimes some arthritic changes; MRI gives the best diagnostic details as demonstrating the bony edema in the distal clavicle, fluid in the acromioclavicular joint and cysts or erosions within the distal clavicle [44]. In Figure 10, a typical MRI slice of DCO is given. Fracture, gout, rheumatoid diseases, hyperparathyroidism, and Gorham's massive osteolysis count for differential diagnosis. The main treatment based on conservative methods as rest, NSAI, magnetic field therapy, activity modifications, and ice; if it is refractory to all, surgical treatment is indicated as excision of distal clavicle.

\section{Conclusion}

Clavicle fractures are one of the most frequent fractures and although most of them can be treated with various conservative methods, surgical treatment among in athletes and active young patients is getting more popular. The ideas and treatment methods given in this review can be utilized in a patient who seeks for a faster recovery. Never forget that dealing with an infected non-union of a previously operated clavicle is one of the worst scenarios an orthopedic surgeon may experience. 


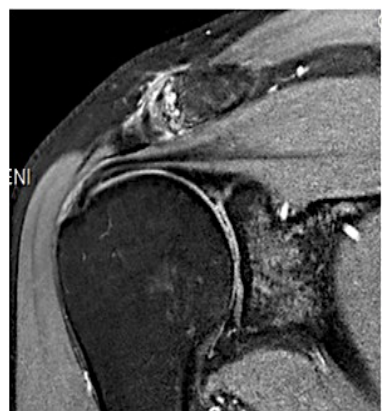

Figure 10: A typical MRI slice of distal clavicle osteolysis.

\section{References}

1. Postacchini F, Gumina S, De Santis P, Albo F (2002) Epidemiology of clavicle fractures. J Shoulder Elbow Surg 11: 452-456.

2. Zhu X, Li W, Chen Z (2004) Analyses of epidemiology in 363 cases of clavicle fractures. Zhongguo Xiu Fu Chong Jian Wai Ke Za Zhi 18: 275-276.

3. McKee MD (2010) Clavicle fractures in 2010: sling/swathe or open reduction and internal fixation Orthop Clin North Am 41: 225-231.

4. 4.van der Meijden OA, Gaskill TR, Millett PJ (2012) Treatment of clavicle fractures: current concepts review. J Shoulder Elbow Surg 21: 423-429.

5. Robinson CM (1998) Fractures of the clavicle in the adult. Epidemiology and classification. J Bone Joint Surg Br 80: 476-484.

6. Khan LA, Bradnock TJ, Scott C, Robinson CM (2009) Fractures of the clavicle. J Bone Joint Surg Am 91: 447-460.

7. NEER CS 2nd (1960) Nonunion of the clavicle. J Am Med Assoc 172: 1006 1011.

8. Jones GL, McCluskey GM 3rd, Curd DT (2000) Nonunion of the fractured clavicle: evaluation, etiology, and treatment. J South Orthop Assoc 9: 43-54.

9. Ozler T, GÃven M, Kocadal AO, UluÃßay C, BeyzadeoÄŸlu T, et al. (2012) Locked anatomic plate fixation in displaced clavicular fractures. Acta Orthop Traumatol Turc 46: 237-242.

10. Singh R, Rambani R, Kanakaris N, Giannoudis PV (2012) A 2-year experience, management and outcome of 200 clavicle fractures. Injury 43: 159-163.

11. Sharr JR, Mohammed KD (2003) Optimizing the radiographic technique in clavicular fractures. J Shoulder Elbow Surg 12: 170-172.

12. Franck WM, Jannasch O, Siassi M, Hennig FF (2003) Balser plate stabilization: an alternate therapy for traumatic sternoclavicular instability. J Shoulder Elbow Surg 12: 276-281.

13. Schulz R, Mühler M, Mutze S, Schmidt S, Reisinger W, et al. (2005) Studies on the time frame for ossification of the medial epiphysis of the clavicle as revealed by CT scans. Int J Legal Med 119: 142-145.

14. Faraud A, Bonnevialle N, Allavena $C$, Nouaille Degorce $H$, Bonnevialle $P$, et al (2014) Outcomes from surgical treatment of middle-third clavicle fractures nonunion in adults: a series of 21 cases. Orthop Traumatol Surg Res 100: 171-176.

15. Kim W, McKee MD (2008) Management of acute clavicle fractures. Orthop Clin North Am 39: 491-505, vii.

16. Modi N (2011) Plate fixation of acute midshaft clavicle fractures-Results of 172 cases. Injury Extra 42: 122.

17. Tarng YW, Yang SW, Fang YP, Hsu CJ (2012) Surgical management of uncomplicated midshaft clavicle fractures: a comparison between titanium elastic nails and small reconstruction plates. See comment in J Shoulder Elbow Surg 21: 732-740.

18. Jiang H, Qu W (2012) Operative treatment of clavicle midshaft fractures using a locking compression plate: comparison between mini-invasive plate osteosynthesis (MIPPO) technique and conventional open reduction. Orthop Traumatol Surg Res 98: 666-671.

19. Collinge C, Devinney S, Herscovici D, DiPasquale T, Sanders R (2006)
Anterior-inferior plate fixation of middle-third fractures and nonunions of the clavicle. J Orthop Trauma 20: 680-686.

20. Lee HJ, Oh CW, Oh JK, Yoon JP, Kim JW, et al. (2013) Percutaneous plating for comminuted midshaft fractures of the clavicle: a surgical technique to aid the reduction with nail assistance. Injury 44: 465-470.

21. Holst J (2013) Risk Factors for Nonunion after Nonoperative Treatment of Displaced Midshaft Fractures of the Clavicle. The Journal of Emergency Medicine 45: 635

22. Singh AK (2011) Clavicle midshaft fracture non-union treated surgically, with bone grafting or demineralised bone matrix: A comparative outcome analysis. Injury Extra 42: 120.

23. Fu TH, Tan BL, Liu HC, Wang JW (2012) Anatomical reduction for treatment of displaced midshaft clavicular fractures: Knowles pinning vs. reconstruction plating. Orthopedics 35: e23-30.

24. Smekal V, Irenberger A, Attal RE, Oberladstaetter J, Krappinger D, et al. (2011) Elastic stable intramedullary nailing is best for mid-shaft clavicular fractures without comminution: results in 60 patients Injury 42: 324-329.

25. Koehler DM, Shipman J, Davidson MA, Guillamondegui O (2011) Is early venous thromboembolism prophylaxis safe in trauma patients with intracranial hemorrhage. J Trauma 70: 324-329.

26. Bonnevialle N, Delannis Y, Mansat P, Peter O, Chemama B, et al. (2010) Bilateral clavicle fracture external fixation. Orthop Traumatol Surg Res 96 : 821-824.

27. Bahk MS, Kuhn JE, Galatz LM, Connor PM, Williams GR Jr (2009) Acromioclavicular and sternoclavicular injuries and clavicular, glenoid, and scapular fractures. J Bone Joint Surg Am 91: 2492-2510.

28. Xin Gu, Biao Cheng, Jian Sun, Kun Tao (2012) Clavicular Hook Plate: A Better Implant Choice for Fixation of Unstable Distal Clavicle Fractures? Journal of Experimental \& Clinical Medicine 4: 270-274.

29. Madsen W, Yaseen Z, LaFrance R, Chen T, Awad H, et al. (2013) Addition of a suture anchor for coracoclavicular fixation to a superior locking plate improves stability of type IIB distal clavicle fractures. Arthroscopy 29: 998-1004.

30. Motta, Bruno L, Maderni A, Tosco P, Mariotti U(2014) Acute lateral dislocated clavicular fractures: arthroscopic stabilization with TightRope. J Shoulder Elbow Surg 23: e47-52.

31. Shin SJ, Roh KJ, Kim JO, Sohn HS (2009) Treatment of unstable distal clavicle fractures using two suture anchors and suture tension bands. Injury 40 : 1308-1312.

32. Kalamaras M, Cutbush K, Robinson M (2008) A method for internal fixation of unstable distal clavicle fractures: early observations using a new technique. J Shoulder Elbow Surg 17: 60-62.

33. Flinkkilä T, Ristiniemi J, Hyvönen $P$, Hämäläinen $M$ (2002) Surgical treatment of unstable fractures of the distal clavicle: a comparative study of Kirschner wire and clavicular hook plate fixation.Acta Orthop Scand 73: 50-53.

34. Meda PV, Machani B, Sinopidis C, Braithwaite I, Brownson P, et al. (2006) Clavicular hook plate for lateral end fractures:- a prospective study.Injury 37 277-283.

35. Esenyel CZ, Ceylan HH, AyanoÄŸlu S, Kebudi A, Adanir O, et al. (2011) Treatment of Neer Type 2 fractures of the distal clavicle with coracoclavicular screw. Acta Orthop Traumatol Turc 45: 291-296.

36. Balcik BJ, Monseau AJ, Krantz W (2013) Evaluation and treatment of sternoclavicular, clavicular, and acromioclavicular injuries. Prim Care 40: 911-923

37. Beris A, Lykissas M, Kostas-Agnantis I, Vekris M, Mitsionis G, et al. (2013) Management of acute acromioclavicular joint dislocation with a double-button fixation system. Injury 44: 288-292.

38. Bontempo NA, Mazzocca AD (2010) Biomechanics and treatment of acromioclavicular and sternoclavicular joint injuries. $\mathrm{Br} \mathrm{J}$ Sports Med 44: 361-369.

39. Johansen JA, Grutter PW, McFarland EG, Petersen SA (2011) Acromioclavicula joint injuries: indications for treatment and treatment options. J Shoulder Elbow Surg 20: S70-82

40. Beitzel K, et al. (2013) Current Concepts in the Treatment of Acromioclavicular 
Citation: Cagatay U, Altintas F, Ozler T, Aksu B (2015) Treatment of Clavicle Fractures in Athletes and Young Active Patients. J Trauma Treat 4: 237. doi:10.4172/2167-1222.1000237

Page 6 of 6

Joint Dislocations. Arthroscopy: The Journal of Arthroscopic \& Related Surgery. 29: 387-397

41. Dabis J, Chakravarthy J, Kalogrianitis S (2011) Management of Grade III acromioclavicular joint dislocation using arthroscopic tight rope fixation. Injury Extra 42: 123

42. Choi SH, Choi GH, Han DH, Choi JS (2015) Laparoscopic liver resection using a rubber band retraction technique: usefulness and perioperative outcome in 100 consecutive cases.Surg Endosc 29: 387-397.

43. DeBerardino TM, Pensak MJ, Ferreira J, Mazzocca AD (2010) Arthroscopic stabilization of acromioclavicular joint dislocation using the AC graftrope system. J Shoulder Elbow Surg 19: 47-52.

44. Wright AP, MacLeod IAR, Talwalker SC (2011) Disorders of theacromioclavicular joint and distal clavicle. Orthopaedics and Trauma. 